\title{
A Wiener-Hopf Dynamical System for Mixed Equilibrium Problems
}

\author{
Farhat Suhel, ${ }^{1}$ S. K. Srivastava, ${ }^{1}$ and Suhel Ahmad Khan ${ }^{2}$ \\ ${ }^{1}$ Department of Mathematics and Statistics, DDU Gorakhpur University, Gorakhpur 273009, India \\ ${ }^{2}$ Department of Mathematics, BITS Pilani, Dubai Campus, Dubai 345055, UAE
}

Correspondence should be addressed to Farhat Suhel; farhat.math@gmail.com

Received 30 January 2014; Revised 31 March 2014; Accepted 1 April 2014; Published 22 April 2014

Academic Editor: Nawab Hussain

Copyright (C) 2014 Farhat Suhel et al. This is an open access article distributed under the Creative Commons Attribution License, which permits unrestricted use, distribution, and reproduction in any medium, provided the original work is properly cited.

We suggest and analyze dynamical systems associated with mixed equilibrium problems by using the resolvent operator technique. We show that these systems have globally asymptotic property. The concepts and results presented in this paper extend and unify a number of previously known corresponding concepts and results in the literature.

\section{Introduction}

Equilibrium problems theory has emerged as an interesting and fascinating branch of applicable mathematics. This theory has become a rich source of inspiration and motivation for the study of a large number of problems arising in economics, optimization, and operation research in a general and unified way. There are a substantial number of papers on existence results for solving equilibrium problems based on different-relaxed monotonicity notions and various compactness assumptions; see, for example, [1-6]. In 2002, Moudafi [5] considered a class of mixed equilibrium problems which includes variational inequalities as well as complementarity problems, convex optimization, saddle point problems, problems of finding a zero of a maximal monotone operator, and Nash equilibria problems as special cases. He studied sensitivity analysis and developed some iterative methods for mixed equilibrium problems. In recent years, much attention has been given to consider and analyze the projected dynamical systems associated with variational inequalities and nonlinear programming problems, in which the right-hand side of the ordinary differential equation is a projection operator. Such types of the projected dynamical system were introduced and studied by Dupuis and Nagurney [7]. Projected dynamical systems are characterized by a discontinuous right-hand side. The discontinuity arises from the constraint governing the question. The innovative and novel feature of a projected dynamical systems is that the set of stationary points of dynamical system correspond to the set of solution of the variational inequality problems. It has been shown in [8-14] that the dynamical systems are useful in developing efficient and powerful numerical technique for solving variational inequalities and related optimization problems. Xia and Wang [13], Zhang and Nagurney [14], and Nagurney and Zhang [11] have studied the globally asymptotic stability of these projected dynamical systems. Noor [15-17] has also suggested and analyzed similar resolvent dynamical systems for variational inequalities. It is worth mentioning that there is no such type of the dynamical systems for mixed equilibrium problems.

In this paper, we show that such type of dynamical systems can be suggested for the mixed equilibrium problems. We consider a mixed equilibrium problem and give its related Wiener-Hopf equation and fixed point formulation. Using this fixed point formulation and Wiener-Hopf equation, we suggest dynamical systems associated with mixed equilibrium problems. We use these dynamical systems to prove the uniqueness of a solution of mixed equilibrium problems. Further, we show that the dynamical systems have globally asymptotic stability property. Our results can be viewed as significant and unified extensions of the known results in this area; see, for example, [6, 13, 15-17]. 


\section{Formulation and Basic Facts}

Let $\mathbb{R}^{n}$ be an Euclidean space, whose inner product and norm are denoted by $\langle\cdot, \cdot\rangle$ and $\|\cdot\|$, respectively. Let $K$ be a nonempty closed convex set in $\mathbb{R}^{n}$, let $T, A: K \rightarrow K$ be nonlinear mappings, and let $N: K \times K \rightarrow K$ be a nonlinear mapping, if $F: K \times K \rightarrow \mathbb{R}$ is a given bifunction satisfying $F(x, x)=0$ for all $x \in K$. Consider the following mixed equilibrium problem (for short MEP): find $x \in K$ such that

$$
F(x, y)+\langle N(T x, A x), y-x\rangle \geq 0, \quad \forall y \in K
$$

This problem has potential and useful applications in nonlinear analysis and mathematical economics. For example, if we set $F(x, y)=\phi(y)-\phi(x)$, for all $x, y \in K$, $\phi: K \rightarrow \mathbb{R}$, a real-valued function, and $N=0$, then MEP (1) reduces to the following minimization problem subject to implicit constraints:

find $x \in K$ such that $\phi(x) \leq \phi(y), \quad \forall y \in K$.

The basic case of variational inclusions corresponds to $F(x, y)=\sup _{w \in B x}\langle w, y-x\rangle$ with $B: K \rightarrow 2^{K}$, a set-valued maximal monotone operator. Actually, MEP (1) is equivalent to the following: find $x \in K$ such that

$$
0 \in N(T x, A x)+B x, \quad \forall y \in K
$$

Moreover, if $F(x, y)=\phi(y)-\phi(x)$, then inclusion (3) reduces to find $x \in K$ such that

$$
\phi(y)-\phi(x)+\langle N(T x, A x), y-x\rangle \geq 0, \quad \forall y \in K .
$$

In particular if $\phi=0, N(T x, A x)=S x$ for all $x \in K$, where $S: K \rightarrow K$, and $K$ is a closed and convex cone, then inequality (4) can be written as

$$
\text { find } x \in K \text { such that } S x \in K^{*}, \quad\langle S x, x\rangle=0 \text {, }
$$

where $K^{*}=\left\{x \in \mathbb{R}^{n}:\langle x, y\rangle \geq 0\right.$, for all $\left.y \in K\right\}$ is the polar cone to $K$. The problem of finding such $x$ is an important instance of well-known complementarity problem of mathematical programming.

Another example corresponds to Nash equilibria in noncooperative games. Let $I$ (the set of players) be a finite index set. For every $i \in I$, let $K_{i}$ (the strategy set of $i$ th player) be a given set, $f_{i}$ (the loss function of the $i$ th player, defending on the strategies of all players): $K \rightarrow \mathbb{R}$ a given function with $K:=\prod_{i \in I} K_{i}$. For $x=\left(x_{i}\right)_{i \in I} \in K$, we define $x^{i}:=\left(x_{j}\right)_{j \in I}$, $j \neq i$. The point $x=\left(x_{i}\right)_{i \in I} \in K$ is called Nash equilibrium if and only if for all $i \in I$ the following inequalities hold true:

$$
f_{i}(x) \leq f_{i}\left(x^{i}, y_{i}\right), \quad \forall y_{i} \in K_{i}
$$

(i.e., no player can reduce his loss by varying his strategy alone). Let $N=0$ and define $F: K \times K \rightarrow \mathbb{R}$ by $F(x, y)=$ $\sum_{i \in I}\left(f_{i}\left(x^{i}, y_{i}\right)-f_{i}(x)\right)$. Then $x \in K$ is a Nash equilibrium if and only if $x$ solves MEP (1).

The following definitions and theorem will be needed in the sequel.
Definition 1 (see [14]). Let $F: K \times K \rightarrow \mathbb{R}$ be a real-valued function. Then $F$ is said to be

(a) monotone if $F(x, y)+F(y, x) \leq 0$, for each $x, y \in K$;

(b) strictly monotone if $F(x, y)+F(y, x)<0$, for each $x, y \in K$, with $x \neq y$;

(c) upper hemicontinuous, if, for all $x, y, z \in K$, $\limsup _{t \rightarrow 0^{+}} F(t z+(1-t) x, y) \leq F(x, y)$.

Theorem 2 (see [14]). If the following conditions hold true for $F: K \times K \rightarrow \mathbb{R}:$

(i) F is monotone and upper hemicontinuous,

(ii) $F(x, \cdot)$ is convex and lower semicontinuous for each $x \in$ $K$,

(iii) there exists a compact subset $B$ of $\mathbb{R}^{n}$ and there exists $y_{0} \in B \cap K$ such that $F\left(x, y_{0}\right)<0$ for each $x \in K \backslash B$,

then the set of solutions to the equilibrium problem

$$
\text { find } x \in K \text { such that } F(x, y) \geq 0, \quad \forall y \in K \text {, }
$$

is nonempty convex and compact. Moreover, if $F$ is strictly monotone, then the solution of equilibrium problem is unique.

Let us recall the extension of the Yosida approximation notion introduced in [5]. Let $\mu>0$, for a given bifunction $F$; the associated Yosida approximation, $F_{\mu}$, over $K$ and the corresponding regularized operator, $A_{\mu}^{F}$, are defined as follows:

$$
\begin{aligned}
F_{\mu}(x, y) & =\left\langle\frac{1}{\mu}\left(x-J_{\mu}^{F}(x)\right), y-x\right\rangle, \\
A_{\mu}^{F} & :=\frac{1}{\mu}\left(x-J_{\mu}^{F}(x)\right),
\end{aligned}
$$

in which $J_{\mu}^{F}(x) \in K$ is the unique solution of

$$
\mu F\left(J_{\mu}^{F}(x), y\right)+\left\langle J_{\mu}^{F}(x)-x, y-J_{\mu}^{F}(x)\right\rangle \geq 0, \quad \forall y \in K .
$$

Remark 3 (see [5]). (i) The existence and uniqueness of the solution of problem (9) follow by invoking Theorem 2 .

(ii) If $F(x, y)=\sup _{u \in B x}\langle u, y-x\rangle$ and $K=\mathbb{R}^{n}, B$ being a maximal monotone operator, it directly yields

$$
J_{\mu}^{F}(x)=(I+\mu B)^{-1} x, \quad A_{\mu}^{F}(x)=B_{\mu}(x),
$$

where $B_{\mu}:=(1 / \mu)\left(I-(I+\mu B)^{-1}\right)$ is the Yosida approximation of $B$, and one recovers classical concepts.

(iii) The operator $J_{\mu}^{F}$ is cocoercive and nonexpansive.

Lemma 4. Assume that conditions of Theorem 2 are fulfilled; then the operator $J_{\mu}^{F}$ is cocoercive with modulus 1; that is,

$$
\begin{array}{r}
\left\langle J_{\mu}^{F}(x)-J_{\mu}^{F}(y), x-y\right\rangle \geq\left|J_{\mu}^{F}(x)-J_{\mu}^{F}(y)\right|^{2}, \\
\forall x, y \in K .
\end{array}
$$


$J_{\mu}^{F}$ is 1-firmly nonexpansive, that is,

$$
\left|J_{\mu}^{F}(x)-J_{\mu}^{F}(y)\right|^{2} \leq|x-y|^{2}-\left|\left(I-J_{\mu}^{F}\right) x-\left(I-J_{\mu}^{F}\right) y\right|^{2},
$$

and $A_{\mu}^{F}$ is cocoercive with modulus $\mu$, that is,

$$
\begin{array}{r}
\left\langle A_{\mu}^{F}(x)-A_{\mu}^{F}(y), x-y\right\rangle \geq \mu\left|A_{\mu}^{F}(x)-A_{\mu}^{F}(y)\right|^{2} \\
\forall x, y \in K .
\end{array}
$$

Proof. From the relation (9), we can write

$$
\begin{array}{r}
\mu F\left(J_{\mu}^{F}(x), J_{\mu}^{F}(y)\right)+\left\langle J_{\mu}^{F}(x)-x, J_{\mu}^{F}(y)-J_{\mu}^{F}(x)\right\rangle \geq 0, \\
\forall x, y \in K, \\
\mu F\left(J_{\mu}^{F}(y), J_{\mu}^{F}(x)\right)+\left\langle J_{\mu}^{F}(y)-y, J_{\mu}^{F}(x)-J_{\mu}^{F}(y)\right\rangle \geq 0, \\
\forall x, y \in K .
\end{array}
$$

By adding the last two inequalities and using the monotonicity of $F$, we obtain the desired result. sively

Equation (12) follows from (11); indeed we have succes-

$$
\begin{aligned}
& \left|\left(I-J_{\mu}^{F}\right) x-\left(I-J_{\mu}^{F}\right) y\right|^{2} \\
& =|x-y|^{2}-2\left\langle J_{\mu}^{F}(x)-J_{\mu}^{F}(y), x-y\right\rangle \\
& \quad+\left|J_{\mu}^{F}(x)-J_{\mu}^{F}(y)\right|^{2} \\
& \leq|x-y|^{2}-\left|J_{\mu}^{F}(x)-J_{\mu}^{F}(y)\right|^{2} .
\end{aligned}
$$

Now combining (11) with $x=J_{\mu}^{F}(x)+\lambda A_{\mu}^{F}(x)$ and $y=J_{\mu}^{F}(y)+$ $\lambda A_{\mu}^{F}(y)$, we obtain

$$
\left\langle A_{\mu}^{F}(x)-A_{\mu}^{F}(y), J_{\mu}^{F}(x)-J_{\mu}^{F}(y)\right\rangle \geq 0 .
$$

On the other hand

$$
\begin{aligned}
\left\langle A_{\mu}^{F}(x)-A_{\mu}^{F}(y), x-y\right\rangle & \\
= & \left\langle A_{\mu}^{F}(x)-A_{\mu}^{F}(y), x-J_{\mu}^{F}(x)-\left(y-J_{\mu}^{F}(y)\right)\right\rangle \\
& \quad+\left\langle A_{\mu}^{F}(x)-A_{\mu}^{F}(y), J_{\mu}^{F}(x)-J_{\mu}^{F}(y)\right\rangle .
\end{aligned}
$$

The announced result follows by noticing that

$$
\begin{aligned}
& \left\langle A_{\mu}^{F}(x)-A_{\mu}^{F}(y), x-J_{\mu}^{F}(x)-\left(y-J_{\mu}^{F}(y)\right)\right\rangle \\
& =\mu\left|A_{\mu}^{F}(x)-A_{\mu}^{F}(y)\right|^{2} .
\end{aligned}
$$

Lemma 5. MEP (1) has a solution $x$ if and only if $x$ satisfies

$$
x=J_{\mu}^{F}(x-\mu N(T x, A x)), \quad \text { for } \mu>0 .
$$

Proof. Let $x \in K$ be a solution of MEP (1); then

$$
F(x, y)+\langle N(T x, A x), y-x\rangle \geq 0, \quad \forall y \in K,
$$

which can be written as

$$
\mu F(x, y)+\langle\mu N(T x, A x), y-x\rangle \geq 0,
$$

where $\mu>0$ is a constant. Thus, for all $x \in K$, we have

$$
\mu F(x, y)+\langle x-(x-\mu N(T x, A x)), y-x\rangle \geq 0,
$$

which is equivalent to

$$
x=J_{\mu}^{F}(x-\mu N(T x, A x)),
$$

by Lemma 4 . This completes the proof.

We now define the residue vector $R(x)$ by the relation

$$
R(x)=x-J_{\mu}^{F}[x-\mu N(T x, A x)] .
$$

Invoking Lemma 5, one can observe that $x \in K$ is a solution of MEP (1) if and only if $x \in K$ is a zero of

$$
R(x)=0 .
$$

Now related to MEP (1), we consider the following Wiener-Hopf equation (in short, WHE): find $z \in \mathbb{R}^{n}$ such that, for $x \in K$,

$$
\begin{aligned}
& N(T x, A x)+A_{\mu}^{F}(z)=0, \\
& x=J_{\mu}^{F}(z), \quad \text { for } \mu>0 .
\end{aligned}
$$

Lemma 6. MEP (1) has a solution $x$ if and only if WHE (26) has a solution $z \in \mathbb{R}^{n}$ where

$$
\begin{gathered}
x=J_{\mu}^{F}(z), \\
z=x-\mu N(T x, A x), \quad \text { for } \mu>0 .
\end{gathered}
$$

Using (27), WHE (26) can be written as

$$
\begin{gathered}
x-\mu N(T x, A x)-J_{\mu}^{F}[x-\mu N(T x, A x)] \\
+\mu N\left(T\left(J_{\mu}^{F}[x-\mu N(T x, A x)]\right),\right. \\
\left.A\left(J_{\mu}^{F}[x-\mu N(T x, A x)]\right)\right)=0 .
\end{gathered}
$$

Thus it is clear from Lemma 6 that $x \in K$ is a solution of $M E P(1)$ if and only if $x \in K$ satisfies (28). 
Using this equivalence, we suggest a new dynamical system associated with MEP (1) as

$$
\begin{array}{r}
\frac{d x}{d t}=\lambda\left\{J_{\mu}^{F}[x-\mu N(T x, A x)]\right. \\
-\mu N\left(T\left(J_{\mu}^{F}[x-\mu N(T x, A x)]\right),\right. \\
\left.A\left(J_{\mu}^{F}[x-\mu N(T x, A x)]\right)\right) \\
+\mu N(T x, A x)-x\}, \\
=\lambda\{-R(x)+\mu N(T x, A x) \\
-\mu N\left(T\left(J_{\mu}^{F}[x-\mu N(T x, A x)]\right),\right. \\
\left.A\left(J_{\mu}^{F}[x-\mu N(T x, A x)]\right)\right\},
\end{array}
$$

$x\left(t_{0}\right)=x_{0} \in K$,

where $\lambda$ is a constant. The system of type (29) is called the resolvent dynamical system associated with mixed equilibrium problem (29) (in short, RDS-MEP). Here the right-hand side is associated with resolvent and hence is discontinuous on the boundary of $K$. It is clear from the definitions that the solution to (29) belongs to the constraints set $K$. This implies that the results such as the existence, uniqueness, and continuous dependence on the given data can be studied. It is worth mentioning that RDS-MEP (29) is different from one considered and studied in [15-17]. sequel.

The following concepts and results are useful in the

Definition 7. The dynamical system is said to converge to the solution set $K^{*}$ of MEP (1) if and only if, irrespective of the initial point, the trajectory of the dynamical system satisfies

$$
\lim _{t \rightarrow \infty} \operatorname{dist}\left(x(t), K^{*}\right),
$$

where

$$
\operatorname{dist}\left(x, K^{*}\right)=\inf _{y \in K^{*}}\|x-y\|
$$

It is easy to see that if the set $K^{*}$ has a unique point $x^{*}$, then (30) implies that $\lim _{t \rightarrow \infty} x(t)=x^{*}$.

If the dynamical system is still stable at $x^{*}$ in the Lyapunov sense, then the dynamical system is globally asymptotically stable at $x^{*}$.

Definition 8. The dynamical system is said to be globally exponentially stable with degree $\eta$ at $x^{*}$ if and only if, irrespective of the initial point, the trajectory of the system $x(t)$ satisfies

$$
\left\|x(t)-x^{*}\right\| \leq \mu_{1}\left\|x\left(t_{0}\right)-x^{*}\right\| \exp \left(-\eta\left(t-t_{0}\right)\right), \quad \forall t \geq t_{0},
$$

where $\mu_{1}$ and $\eta$ are positive constants independent of the initial point. It is clear that globally exponential stability is necessarily globally asymptotical stability and the dynamical system converges arbitrarily fast.

Lemma 9 (Gronwall; see [9]). Let $\bar{x}$ and $\bar{y}$ be real-valued nonnegative continuous function with domain $\left\{t: t \leq t_{0}\right\}$ and let $\alpha(t)=\alpha_{0}\left(\left|t-t_{0}\right|\right)$, where $\alpha_{0}$ is a monotone increasing function. If, for $t \geq t_{0}$,

$$
\bar{x} \leq \alpha(t)+\int_{t_{0}}^{t} \bar{x}(s) \bar{y}(s) d s,
$$

then

$$
\bar{x}(s) \leq \alpha(t)+\exp \left(\left\{\int_{t_{0}}^{t} \bar{y}(s) d s\right\}\right) .
$$

In the sequel, one assumes that the bifunction $F$ involved in MEP (1) satisfies conditions of Theorem 2. Further, from now onward one assumes that $K^{*}$ is nonempty and is bounded, unless otherwise specified. Furthermore, assume that, for all $x \in K$, there exists a constant $\tau>0$ such that

$$
\|N(T x, A x)\| \leq \tau(\|T x\|+\|A x\|) .
$$

We study some properties of RDS-MEP (29) and analyze the global stability of the system. First of all, we discuss the existence and uniqueness of RDS-MEP (29).

\section{Existence and Uniqueness of Solution}

First, we define the following concepts.

Definition 10. Let T, $A: K \rightarrow K, F: K \times K \rightarrow \mathbb{R}$, and $N$ : $K \times K \rightarrow K$ be nonlinear mappings. Then, for all $x, y, z, w \in$ $K$,

(a) $T$ is $\delta$-Lipschitz continuous if there exists a constant $\delta>0$ such that

$$
\|T x-T y\| \leq\|x-y\|
$$

(b) $N$ is $(\alpha, \beta)$-Lipschitz continuous if there exist constants $\alpha, \beta>0$ such that

$$
\|N(x, y)-N(z, w)\| \leq \alpha\|x-z\|+\beta\|y-w\|
$$

(c) $N$ is mixed monotone with respect to $T$ and $A$, if

$$
\langle N(T x, A x)-N(T y, A y), x-y\rangle \geq 0
$$


(d) $F$ is said to be $\theta$-pseudomonotone, where $\theta$ is a realvalued multivariate function, if

$$
F(x, y)+\theta \geq 0 \quad \text { implies }-F(y, x)+\theta \geq 0
$$

Theorem 11. Let the mappings T, $A$, and $N$ be $\delta$-Lipschitz continuous, $\gamma$-Lipschitz continuous, and $(\alpha, \beta)$-Lipschitz continuous, respectively. For each $x_{0} \in \mathbb{R}^{n}$, there exists a unique continuous solution $x(t)$ of RDS-MEP (29) with $x\left(t_{0}\right)=t_{0}$ over $\left[t_{0}, \infty\right)$.

Proof. Let

$$
\begin{gathered}
G(x)=\lambda\left\{J_{\mu}^{F}[x-\mu N(T x, A x)]\right. \\
-\mu N\left(T\left(J_{\mu}^{F}[x-\mu N(T x, A x)]\right),\right. \\
\left.A\left(J_{\mu}^{F}[x-\mu N(T x, A x)]\right)\right) \\
+\mu N(T x, A x)-x\},
\end{gathered}
$$

where $\lambda$ is a constant. For all $x, y \in \mathbb{R}^{n}$, we have

$$
\begin{aligned}
& \|G(x)-G(y)\| \\
& \leq \lambda\left\{\left\|J_{\mu}^{F}[x-\mu N(T x, A x)]-J_{\mu}^{F}[y-\mu N(T y, A y)]\right\|\right. \\
& +\mu\|N(T x, A x)-N(T y, A y)\|+\|x-y\| \\
& +\mu \| N\left(T\left(J_{\mu}^{F}[x-\mu N(T x, A x)]\right)\right. \\
& \left.A\left(J_{\mu}^{F}[x-\mu N(T x, A x)]\right)\right) \\
& -N\left(T\left(J_{\mu}^{F}[y-\mu N(T y, A y)]\right)\right. \\
& \left.\left.A\left(J_{\mu}^{F}[y-\mu N(T y, A y)]\right)\right) \|\right\} \\
& \leq \lambda\{2\|x-y\|+2 \mu\|N(T x, A x)-N(T y, A y)\| \\
& +\mu(\alpha \delta+\beta \gamma) \\
& \times \lambda\{x-y\|+\mu\| N(T x, A x)-N(T y, A y) \|]\} \\
& \left.+3 \mu(\alpha \delta+\beta \gamma)+\mu^{2}(\alpha \delta+\beta \gamma)^{2}\right\}\|x-y\|
\end{aligned}
$$

This implies that the mapping $G$ is Lipchitz continuous in $\mathbb{R}^{n}$. So, for each $x_{0} \in \mathbb{R}^{n}$, there exists a unique and continuous solution $x(t)$ of RDS-MEP (29), defined in an interval $t_{0} \leq t<T$ with initial condition $x\left(t_{0}\right)=x_{0}$. Let $\left[t_{0}, T\right)$ be its maximal interval of existence; we show that $T=$ $\infty$. We estimate

$$
\begin{aligned}
& \|G(x)\|=\lambda \| J_{\mu}^{F}[x-\mu N(T x, A x)] \\
& -\mu N\left(T\left(J_{\mu}^{F}[x-\mu N(T x, A x)]\right),\right. \\
& \left.A\left(J_{\mu}^{F}[x-\mu N(T x, A x)]\right)\right) \\
& +\mu N(T x, A x)-x \| . \\
& \leq \lambda\left\|J_{\mu}^{F}[x-\mu N(T x, A x)]-x\right\| \\
& +\lambda \mu(\alpha \delta+\beta \gamma)\left\|J_{\mu}^{F}[x-\mu N(T x, A x)]-x\right\| \\
& =\lambda(1+\mu(\alpha \delta+\beta \gamma))\left\|J_{\mu}^{F}[x-\mu N(T x, A x)]-x\right\| \\
& \leq \lambda(1+\mu(\alpha \delta+\beta \gamma)) \\
& \times\left\{\left\|J_{\mu}^{F}[x-\mu N(T x, A x)]-J_{\mu}^{F}(x)\right\|\right. \\
& \left.+\left\|J_{\mu}^{F}(x)-J_{\mu}^{F}\left(x^{*}\right)\right\|+\left\|J_{\mu}^{F}\left(x^{*}\right)-x\right\|\right\} \\
& \leq \lambda(1+\mu(\alpha \delta+\beta \gamma)) \\
& \times\left\{\mu\|N(T x, A x)\|+\left\|x-x^{*}\right\|+\left\|J_{\mu}^{F}\left(x^{*}\right)-x\right\|\right\} \\
& \leq \lambda(1+\mu(\alpha \delta+\beta \gamma)) \\
& \times\left\{\mu \tau(\|T x\|+\|A x\|)+\|x\|+\left\|x^{*}\right\|\right. \\
& \left.+\left\|J_{\mu}^{F}\left(x^{*}\right)\right\|+\|x\|\right\}, \\
& \leq \lambda(1+\mu(\alpha \delta+\beta \gamma)) \\
& \times\left\{(\mu \tau(\delta+\gamma)+2)\|x\|+\left\|J_{\mu}^{F}\left(x^{*}\right)\right\|+\left\|x^{*}\right\|\right\} . \\
& =\lambda(1+\mu(\alpha \delta+\beta \gamma))(2+\mu \tau(\delta+\gamma))\|x\| \\
& +\lambda(1+\mu(\alpha \delta+\beta \gamma))\left\{\left\|J_{\mu}^{F}\left(x^{*}\right)\right\|+\left\|x^{*}\right\|\right\},
\end{aligned}
$$

for any $u \in \mathbb{R}^{n}$; then

$$
\begin{aligned}
\|x(t)\| & \leq\left\|x_{0}\right\|+\int_{t_{0}}^{t}\|G x(s)\| d s, \\
& \leq\left(\left\|x_{0}\right\|+k_{1}\left(t-t_{0}\right)\right)+k_{2} \int_{t_{0}}^{t}\|x(s)\| d s,
\end{aligned}
$$

where

$$
\begin{gathered}
k_{1}=\lambda(1+\mu(\alpha \delta+\beta \gamma))\left\{\left\|J_{\mu}^{F}\left(x^{*}\right)\right\|+\left\|x^{*}\right\|\right\}, \\
k_{2}=\lambda(1+\mu(\alpha \delta+\beta \gamma))(2+\mu \tau(\delta+\gamma)) .
\end{gathered}
$$

Therefore, using Lemma 9, we have

$\|x(t)\| \leq\left(\left\|x_{0}\right\|+k_{1}\left(t-t_{0}\right)\right) \exp \left\{k_{2}\left(t-t_{0}\right)\right\}, \quad t \in\left[t_{0}, T\right)$.

Hence, the solution $\|x(t)\|$ is bounded on $\left[t_{0}, T\right)$. So $T=$ $\infty$. This completes the proof. 


\section{Stability Analysis}

We now study the stability of RDS-MEP (29). The analysis is in the spirit of Xia and Wang [13].

Theorem 12. Let the mappings $T, A$, and $N$ be the same as Theorem 11. Let the function $F$ be $\theta$-pseudomonotone with respect to $\theta$, where $\theta$ is defined as

$$
\theta(x, y)=\langle N(T x, A x), y-x\rangle, \quad \forall x, y \in K,
$$

and let $N$ be mixed monotone with respect to $T$ and $A$. If $\mu<1 /(\alpha \delta+\beta \gamma)$, then RDS-MEP (29) is stable in the sense of Lyapunov and globally converges to the solution subset of MEP (1).

Proof. Since the mappings $T, A$, and $N$ are Lipschitz continuous, it follows from Theorem 11 that RDS-MEP (29) has a unique continuous solution $x(t)$ over $\left[t_{0}, T\right)$ for any fixed $x_{0} \in$ $K$. Let $x(t)=x\left(t, t_{0} ; x_{0}\right)$ be the solution of the initial-value problem (29). For a given $x^{*} \in K$, consider the following Lyapunov function:

$$
L(x)=\left\|x-x^{*}\right\|^{2}, \quad x \in \mathbb{R}^{n} .
$$

It is clear that $\lim _{n \rightarrow \infty} L\left(x_{n}\right)=+\infty$, whenever the sequence $\left\{x_{n}\right\} \subset K$ and $\lim _{n \rightarrow \infty} x_{n}=+\infty$. Consequently, we conclude that the level sets of $L$ are bounded. Let $x^{*} \in K$ be a solution of MEP (1); then

$$
F\left(x^{*}, y\right)+\left\langle N\left(T x^{*}, A x^{*}\right), y-x^{*}\right\rangle \geq 0, \quad \forall y \in K .
$$

Since $F$ is $\theta$-pseudomonotone and $N$ is mixed monotone then (48) implies that

$$
\begin{gathered}
-F\left(y, x^{*}\right)+\left\langle N\left(T x^{*}, A x^{*}\right), y-x^{*}\right\rangle \geq 0 \\
-F\left(y, x^{*}\right) \geq-\left\langle N\left(T x^{*}, A x^{*}\right), y-x^{*}\right\rangle \\
\geq-\left\langle N(T y, A y), y-x^{*}\right\rangle .
\end{gathered}
$$

That is,

$$
-F\left(y, x^{*}\right)+\left\langle N(T y, A y), y-x^{*}\right\rangle \geq 0, \quad \forall y \in K .
$$

Taking $y=J_{\mu}^{F}[x-\mu N(T x, A x)]$ in (50), we have

$$
\begin{gathered}
-F\left(J_{\mu}^{F}[x-\mu N(T x, A x)], x^{*}\right) \\
+\left\langle N\left(T J_{\mu}^{F}[x-\mu N(T x, A x)]\right)\right. \\
A\left(J_{\mu}^{F}[x-\mu N(T x, A x)]\right) \\
\left.J_{\mu}^{F}[x-\mu N(T x, A x)]-x^{*}\right\rangle \geq 0 .
\end{gathered}
$$

Setting $y=x^{*}, x=x-\mu N(T x, A x)$, and $J_{\mu}^{F}(x)=J_{\mu}^{F}[x-$ $\mu N(T x, A x)]$ in (9), we have

$$
\begin{aligned}
\mu F\left(J_{\mu}^{F}[x-\mu N(T x, A x)], x^{*}\right) & \\
+\langle & J_{\mu}^{F}[x-\mu N(T x, A x)]-x \\
& +\mu N(T x, A x), x^{*} \\
& \left.\quad-J_{\mu}^{F}[x-\mu N(T x, A x)]\right\rangle \geq 0 .
\end{aligned}
$$

From (51), (52), and (24), we have

$$
\begin{gathered}
\langle-R(x)+\mu N(T x, A x) \\
-\mu N\left(T\left(J_{\mu}^{F}[x-\mu N(T x, A x)]\right),\right. \\
\left.A\left(J_{\mu}^{F}[x-\mu N(T x, A x)]\right)\right), \\
\left.x^{*}-x+R(x)\right\rangle \geq 0,
\end{gathered}
$$

which implies that

$$
\begin{aligned}
& \left\langle x-x^{*}, R(x)-\mu N(T x, A x)\right. \\
& +\mu N\left(T\left(J_{\mu}^{F}[x-\mu N(T x, A x)]\right),\right. \\
& \left.\left.A\left(J_{\mu}^{F}[x-\mu N(T x, A x)]\right)\right)\right\rangle \\
& \geq\|R(x)\|^{2} \\
& -\mu\langle R(x), N(T x, A x) \\
& \quad-N\left(T\left(J_{\mu}^{F}[x-\mu N(T x, A x)]\right),\right. \\
& \left.\left.A \| R\left(J_{\mu}^{F}[x-\mu N(T x, A x)]\right)\right)\right\rangle \\
& \quad \times \| x-J_{\mu}^{F}[x-\mu N(\alpha \delta+\beta \gamma)\|R(x)\| \\
& =(1-\mu(\alpha \delta+\beta \gamma))\|R(x)\|^{2} .
\end{aligned}
$$

Thus, from (29), (47), and (54), we have

$$
\begin{aligned}
\frac{d}{d t} L(x)= & \frac{d L}{d x} \frac{d x}{d t} \\
= & 2 \lambda\left\langle x-x^{*},-R(x)+\mu N(T x, A x)\right. \\
& -\mu N\left(T\left(J_{\mu}^{F}[x-\mu N(T x, A x)]\right),\right. \\
& \left.\left.A\left(J_{\mu}^{F}[x-\mu N(T x, A x)]\right)\right)\right\rangle \\
\leq-2 \lambda(1-\mu(\alpha \delta+\beta \gamma))\|R(x)\|^{2} & \quad \text { for } \mu<\frac{1}{(\alpha \delta+\beta \gamma)} .
\end{aligned}
$$

This implies that $L(x)$ is a global Lyapunov function for RDSMEP (29) which is stable in the sense of Lyapunov. Since $\left\{x(t): t \geq t_{0}\right\} \subset K_{0}$ where $K_{0}=\left\{x \in K: L(x) \leq L\left(x_{0}\right)\right\}$ and the function $L(x)$ is continuously differentiable on the bounded and closed set $K$, it follows from LaSalle's invariance principle [9] that the trajectories $x(t)$ will converge to $\Omega$, the largest invariant subset of the following set:

$$
E=\left\{x \in K: \frac{d x}{d t}=0\right\} .
$$


Note that if $(d L / d t)=0$, then

$$
\left\|u-J_{\mu}^{F}[x-\mu N(T x, A x)]\right\|^{2}=0,
$$

and hence $x$ is an equilibrium point of RDS-MEP (29); that is, $d x / d t=0$.

Conversly, if $(d x / d t)=0$, then it follows that $(d L / d t)=$ 0 .

Thus, we conclude that $E=\{x \in K:(d x / d t)=0\}=K_{0} \cap$ $K^{*}$, which is nonempty, convex, and invariant set contained in the solution set $K^{*}$. So, $\lim _{t \rightarrow \infty} \operatorname{dist}(x(t), E)=0$.

Therefore RDS-MEP (29) converges globally to the solution set of MEP (1). In particular, if we set $E=\left\{x^{*}\right\}$, then

$$
\lim _{t \rightarrow \infty} x(t)=x^{*}
$$

Hence RDS-MEP (29) is globally asymptotically stable. This completes the proof.

Theorem 13. Let the mappings $T, A$, and $N$ be the same as in Theorem 11. If $\lambda<0$, then RDS-MEP (29) converges globally exponentially to the unique solution of MEP (1).

Proof. It follows from Theorem 11 that there exists a unique continuously differentiable solution of RDS-MEP (29) over $\left[t_{0}, \infty\right)$. Then

$$
\begin{gathered}
\frac{d L}{d t}=2 \lambda\left\langle x(t)-x^{*},\right. \\
J_{\mu}^{F}[x(t)-\mu N(T x(t), A x(t))] \\
-\mu N\left(T\left(J_{\mu}^{F}[x-\mu N(T x, A x)]\right),\right. \\
\left.A\left(J_{\mu}^{F}[x-\mu N(T x, A x)]\right)\right) \\
+\mu N(T x(t), A x(t))-x(t)\rangle \\
+2 \lambda\left\|x(t)-x^{*}\right\|^{2} \\
+2 \lambda\left\langle x(t)-x^{*}, J_{\mu}^{F}[x(t)-\mu N(T x(t), A x(t))]\right. \\
-\mu N\left(T\left(J_{\mu}^{F}[x-\mu N(T x, A x)]\right),\right. \\
\left.A\left(J_{\mu}^{F}[x-\mu N(T x, A x)]\right)\right) \\
+\mu N(T x(t), A x(t))-x *\rangle,
\end{gathered}
$$

where $x^{*} \in K$ is the solution of MEP (1). Thus

$$
\begin{aligned}
& x^{*}= J_{\mu}^{F}\left[x^{*}-\mu N\left(T x^{*}, A x^{*}\right)\right] \\
&-\mu N\left(T\left(J_{\mu}^{F}[x-\mu N(T x, A x)]\right),\right. \\
&\left.A\left(J_{\mu}^{F}[x-\mu N(T x, A x)]\right)\right) \\
&+\mu N\left(T x^{*}, A x^{*}\right) .
\end{aligned}
$$

Now, we estimate

$$
\begin{aligned}
& \| J_{\mu}^{F}[x(t)-\mu N(T x(t), A x(t))] \\
& -\mu N\left(T\left(J_{\mu}^{F}[x(t)-\mu N(T x(t), A x(t))]\right),\right. \\
& \left.A\left(J_{\mu}^{F}[x(t)-\mu N(T x(t), A x(t))]\right)\right) \\
& +\mu N(T x(t), A x(t))-J_{\mu}^{F}\left[x^{*}-\mu N\left(T x^{*}, A x^{*}\right)\right] \\
& -\mu N\left(T\left(J_{\mu}^{F}\left[x^{*}-\mu N\left(T x^{*}, A x^{*}\right)\right]\right),\right. \\
& \left.A\left(J_{\mu}^{F}\left[x^{*}-\mu N\left(T x^{*}, A x^{*}\right)\right]\right)\right) \\
& +\mu N\left(T x^{*}, A x^{*}\right) \| \\
& \leq\left\|x(t)-x^{*}\right\| \\
& +2 \mu\left\|N(T x(t), A x(t))-N\left(T x^{*}, A x^{*}\right)\right\| \\
& +\mu \| N\left(T\left(J_{\mu}^{F}[x(t)-\mu N(T x(t), A x(t))]\right),\right. \\
& \left.A\left(J_{\mu}^{F}[x(t)-\mu N(T x(t), A x(t))]\right)\right) \\
& -N\left(T\left(J_{\mu}^{F}\left[x^{*}-\mu N\left(T x^{*}, A x^{*}\right)\right]\right),\right. \\
& \left.A\left(J_{\mu}^{F}\left[x^{*}-\mu N\left(T x^{*}, A x^{*}\right)\right]\right)\right) \| \\
& \leq\left\|x(t)-x^{*}\right\|+2 \mu(\alpha \delta+\beta \gamma)\left\|x(t)-x^{*}\right\| \\
& +\mu(\alpha \delta+\beta \gamma)\left\{\left\|x(t)-x^{*}\right\|+\mu(\alpha \delta+\beta \gamma)\left\|x(t)-x^{*}\right\|\right\} \\
& =\left\{1+3 \mu(\alpha \delta+\beta \gamma)+\mu^{2}(\alpha \delta+\beta \gamma)^{2}\right\}\left\|x(t)-x^{*}\right\| .
\end{aligned}
$$

From (59) and (61), we have

$$
\frac{d}{d t}\left\|x(t)-x^{*}\right\|^{2} \leq 2 \lambda \theta\left\|x(t)-x^{*}\right\|^{2}
$$

where $\theta=\mu(\alpha \delta+\beta \gamma)\{3+\mu(\alpha \delta+\beta \gamma)\}$.

Thus, for $\lambda=-\lambda_{1}$, where $\lambda_{1}$ is a positive constant, we have

$$
\left\|x(t)-x^{*}\right\| \leq\left\|x\left(t_{0}\right)-x^{*}\right\| \exp \left\{-\theta \lambda_{1}\left(t-t_{0}\right)\right\},
$$

which shows that the trajectory of the solution of RDS-MEP (29) converges globally exponentially to the unique solution of MEP (1). This completes the proof.

\section{Conflict of Interests}

The authors declare that there is no conflict of interests regarding the publication of this paper.

\section{Acknowledgment}

Farhat Suhel is thankful to NBHM, Department of Atomic Energy, India, Grant no. NBHM/PDF.2/2013/291, for supporting this research work. 


\section{References}

[1] E. Blum and W. Oettli, "From optimization and variational inequalities to equilibrium problems," The Mathematics Student, vol. 63, no. 1-4, pp. 123-145, 1994.

[2] L.-C. Ceng and J.-C. Yao, "A relaxed extragradient-like method for a generalized mixed equilibrium problem, a general system of generalized equilibria and a fixed point problem," Nonlinear Analysis: Theory, Methods \& Applications, vol. 72, no. 3-4, pp. 1922-1937, 2010.

[3] S. A. Khan, B. S. Lee, and F. Suhel, "Vector mixed quasi complementarity problems in Banach spaces," Mathematical and Computer Modelling, vol. 55, no. 3-4, pp. 983-988, 2012.

[4] S. A. Khan and N. Ahmad, "Existence results for vector mixed quasi-complementarity problems," Journal of Mathematics, vol. 2013, Article ID 204348, 6 pages, 2013.

[5] A. Moudafi, "Mixed equilibrium problems: sensitivity analysis and algorithmic aspect," Computers \& Mathematics with Applications, vol. 44, no. 8-9, pp. 1099-1108, 2002.

[6] Y. Shehu, "Iterative method for fixed point problem, variational inequality and generalized mixed equilibrium problems with applications," Journal of Global Optimization, vol. 52, no. 1, pp. 57-77, 2012.

[7] P. Dupuis and A. Nagurney, "Dynamical systems and variational inequalities," Annals of Operations Research, vol. 44, no. 1-4, pp. 9-42, 1993, Advances in equilibrium modeling, analysis and computation.

[8] J. Dong, D. Zhang, and A. Nagurney, "A projected dynamical systems model of general financial equilibrium with stability analysis," Mathematical and Computer Modelling, vol. 24, no. 2, pp. 35-44, 1996.

[9] T. L. Friesz, D. Bernstein, and R. Stough, "Dynamic systems, variational inequalities and control theoretic models for predicting time-varying urban network flows," Transportation Science, vol. 30, no. 1, pp. 14-31, 1996.

[10] S. Giuffrè, G. Idone, and S. Pia, "Some classes of projected dynamical systems in Banach spaces and variational inequalities," Journal of Global Optimization, vol. 40, no. 1-3, pp. 119-128, 2008.

[11] A. Nagurney and D. Zhang, Projected Dynamical Systems and Variational Inequalities with Applications, Kluwer Academic, Dordrecht, The Netherlands, 1995.

[12] Y. Xia and J. Wang, "A recurrent neural network for solving linear projection equations," Neural Networks, vol. 13, no. 3, pp. 337-350, 2000.

[13] Y. S. Xia and J. Wang, "On the stability of globally projected dynamical systems," Journal of Optimization Theory and Applications, vol. 106, no. 1, pp. 129-150, 2000.

[14] D. Zhang and A. Nagurney, "On the stability of projected dynamical systems," Journal of Optimization Theory and Applications, vol. 85, no. 1, pp. 97-124, 1995.

[15] M. A. Noor, "A Wiener-Hopf dynamical system for variational inequalities," New Zealand Journal of Mathematics, vol. 31, no. 2, pp. 173-182, 2002.

[16] M. A. Noor, "Implicit dynamical systems and quasi variational inequalities," Applied Mathematics and Computation, vol. 134, no. 1, pp. 69-81, 2003.

[17] M. A. Noor, "Implicit resolvent dynamical systems for quasi variational inclusions," Journal of Mathematical Analysis and Applications, vol. 269, no. 1, pp. 216-226, 2002. 


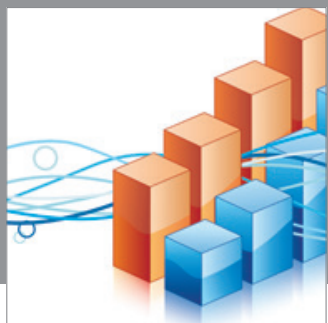

Advances in

Operations Research

mansans

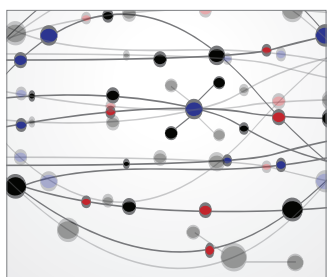

The Scientific World Journal
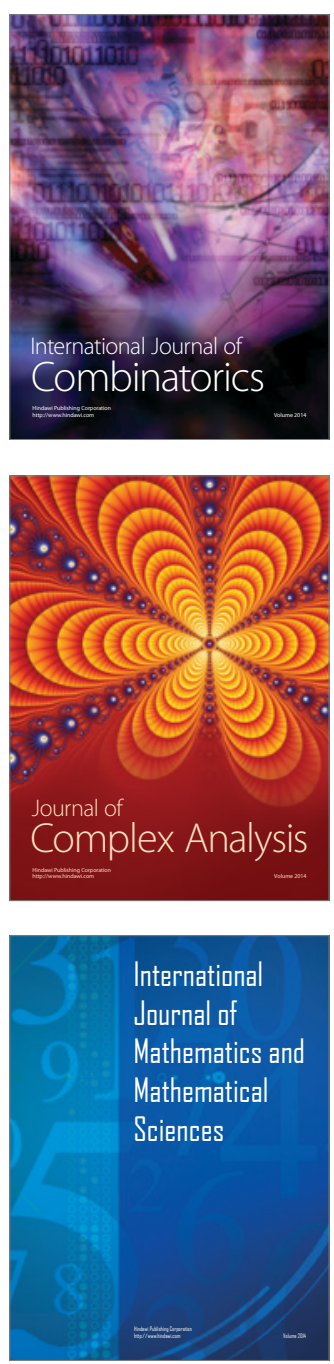
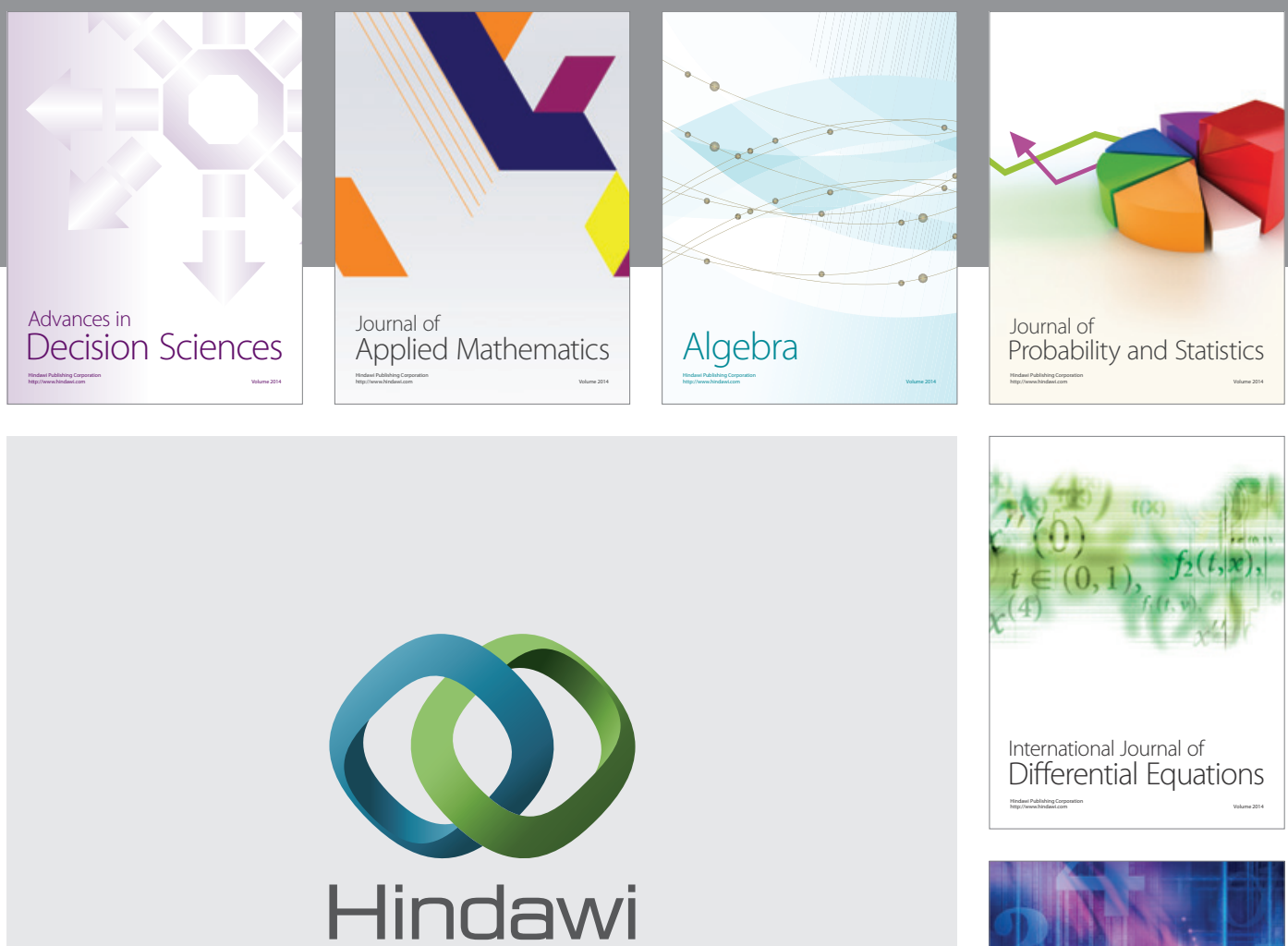

Submit your manuscripts at http://www.hindawi.com
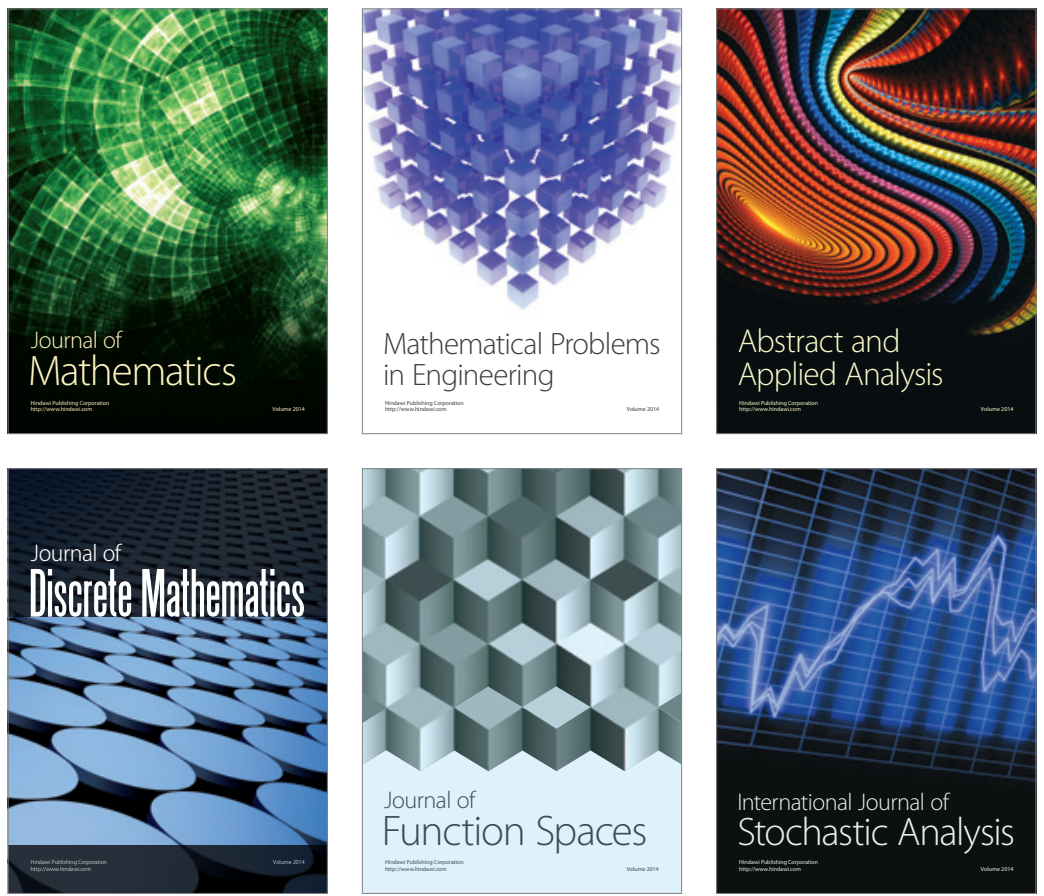

Journal of

Function Spaces

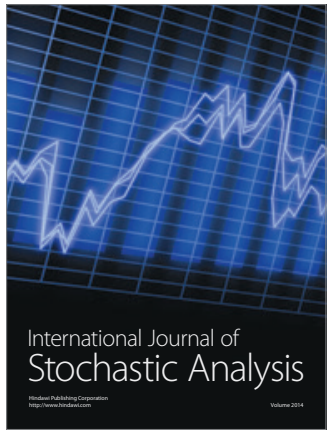

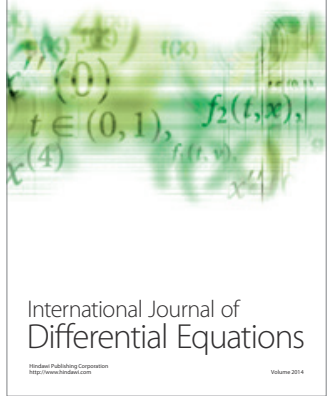
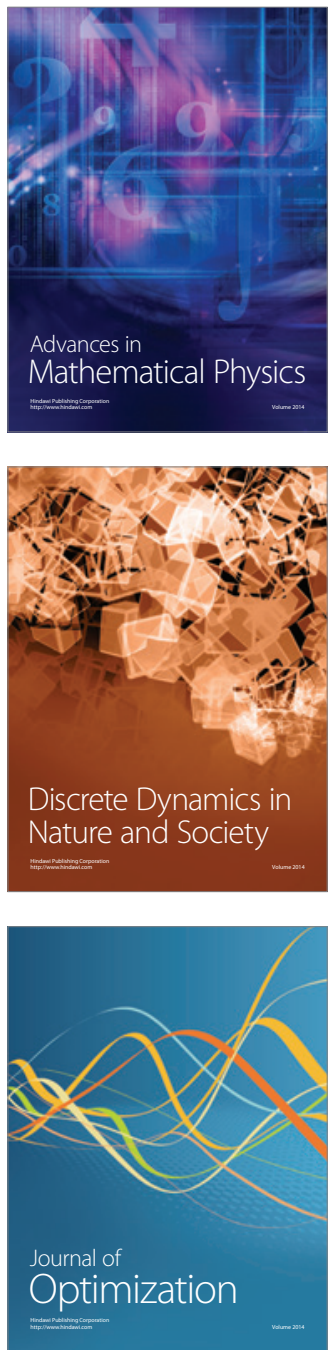